Notre Dame Journal of Formal Logic

Volume 37, Number 4, Fall 1996

\title{
Higman's Embedding Theorem in a General Setting and Its Application to Existentially Closed Algebras
}

\author{
OLEG V. BELEGRADEK
}

\begin{abstract}
For a quasi variety of algebras $\mathbf{K}$, the Higman Theorem is said to be true if every recursively presented $\mathbf{K}$-algebra is embeddable into a finitely presented $\mathbf{K}$-algebra; the Generalized Higman Theorem is said to be true if any $\mathbf{K}$-algebra which is recursively presented over its finitely generated subalgebra is embeddable into a $\mathbf{K}$-algebra which is finitely presented over this subalgebra. We suggest certain general conditions on $\mathbf{K}$ under which (1) the Higman Theorem implies the Generalized Higman Theorem; (2) a finitely generated Kalgebra $A$ is embeddable into every existentially closed $\mathbf{K}$-algebra containing a finitely generated $\mathbf{K}$-algebra $B$ if and only if the word problem for $A$ is $Q$ reducible to the word problem for $B$. The quasi varieties of groups, torsion-free groups, and semigroups satisfy these conditions.
\end{abstract}

1 Neumann [13] showed that every existentially closed group contains a copy of every group with solvable word problem. Macintyre 11 proved the converse: every finitely generated group with unsolvable word problem can be omitted in some existentially closed group. In fact, he proved a more general fact: if $H_{1}$ and $H_{2}$ are finitely generated groups such that the word problem for $H_{1}$ is not Turing reducible to the word problem for $H_{2}$, then $H_{1}$ can be omitted in some existentially closed group containing $\mathrm{H}_{2}$. The result is a special case of an omitting quantifier-free types theorem. It is natural to ask under what conditions the presence of $H_{2}$ in an existentially closed group implies the presence of $H_{1}$. The answer is as follows: $H_{1}$ is embeddable into every existentially closed group containing $\mathrm{H}_{2}$ if and only if the word problem for $\mathrm{H}_{1}$ is $Q$-reducible to the word problem for $\mathrm{H}_{2}$. (The precise definition of the $Q$ reducibility will be given below; for the present, note that this reducibility is stronger than the Turing one.) The result was proved by the author for recursively presented $H_{1}$ and $H_{2}[1]$ and independently by Ziegler [16] in the general case. (The author borrowed the term ' $Q$-reducibility' from Rogers (14], Exercise 9-55) where it was

Received July 31, 1994; revised August 6, 1996 
considered for recursively enumerable sets; Rogers attributed the notion to Tennenbaum. Ziegler called this reducibility the $*$-reducibility.)

We show that the only if part of the theorem above can be proved in a quite general situation. However, in the if part the group theoretic specificity is more essential. A crucial point in the proof is a use of Higman's Embedding Theorem [8] which claims that any recursively presented group can be embedded into a finitely presented group. This theorem suffices to prove the result for recursively presented $H_{1}$ and $H_{2}$, but for arbitrary $H_{1}$ and $H_{2}$ one needs a relativized version of the theorem. Such a generalization of Higman's Embedding Theorem was proved by Ziegler in 16 and C. F. Miller III (unpublished manuscript).

The aim of the present paper is to extend the results above to a more general situation. It does not seem hopeless to search for a generalization of the results because analogues of Higman's Embedding Theorem hold for many natural algebraic classes. Namely, it holds for semigroups (cf. Murskiǔ [12]), inverse semigroups (cf. Belyaev [7]), associative algebras (cf. Belyaev [6]), and Lie algebras (cf. Kukin [10]). Higman's Embedding Theorem also holds for the class of torsion-free groups because this class is closed under operations of free product with amalgamation and HNNextension which are used to construct a finitely presented group containing a given recursively presented group. However, a priori it does not suffice to have an analogue of Higman's Embedding Theorem for a class to prove the result on finitely generated subalgebras of existentially closed algebras in the class. We give some additional conditions on a class which suffice to prove the result as well as a relativized version of Higman's Embedding Theorem for the class. The classes of groups, torsion-free groups, and semigroups satisfy these conditions.

2 Preliminaries Let $U, V \subseteq \omega$. The set $U$ is said to be enumeration reducible to $V$ (in symbols, $U \leq_{e} V$ ) if there is an effective procedure which, for a given enumeration of $V$, produces an enumeration of $U$. A formal definition is as follows (cf. [14], §9.7): $U \leq_{e} V$ if and only if there is a recursively enumerable set $W$ of pairs of the form $(z, Z)$, where $z \in \omega$ and $Z$ is a finite subset of $\omega$ such that $z \in U$ if and only if $(z, Z) \in$ $W$ for some $Z \subseteq V$.

For example, let $\Sigma$ and $\Phi$ be sets of first order $L$-sentences and $\Theta$ the set of all $L$-sentences $\psi$ such that $\Sigma \cup \Phi \vdash \psi$. If $\Sigma$ is recursively enumerable then $\Theta \leq_{e} \Phi$. Indeed, an $L$-sentence $\psi$ is in $\Theta$ if and only if there is a finite $\Psi \subseteq \Phi$ such that $\Sigma \vdash$ $\wedge \Psi \rightarrow \psi$. Let $W$ be the set of pairs $(\psi, \Psi)$ such that $\psi$ is an $L$-sentence, $\Psi$ is a finite set of $L$-sentences, and $\Sigma \vdash \wedge \Psi \rightarrow \psi$. As $\Sigma$ is recursively enumerable, $W$ is recursively enumerable too.

Let $L$ be a recursive functional signature. For a sequence $\bar{a}$ in an $L$-algebra $A$, we denote by $\langle\bar{a}\rangle_{A}$, or simply by $\langle\bar{a}\rangle$, the subalgebra of $A$ generated by $\bar{a}$. The set of all atomic or negated atomic $L(\bar{a})$-formulas which hold in $(A, \bar{a})$ is said to be the diagram of $\bar{a}$ in $A$ and is denoted by $\operatorname{diag}_{A}(\bar{a})$. The set of all atomic $L(\bar{a})$-formulas which hold in $(A, \bar{a})$ is said to be the positive diagram of $\bar{a}$ in $A$ and is denoted by $\operatorname{diag}_{A}^{+}(\bar{a})$. Note that for any tuples $\bar{a}_{1}$ and $\bar{a}_{2}$ in $A$ with $\left\langle\bar{a}_{1}\right\rangle=\left\langle\bar{a}_{2}\right\rangle$, the sets $\operatorname{diag}_{A}\left(\bar{a}_{1}\right)$ and $\operatorname{diag}_{A}\left(\bar{a}_{2}\right)$, as well as $\operatorname{diag}_{A}^{+}\left(\bar{a}_{1}\right)$ and $\operatorname{diag}_{A}^{+}\left(\bar{a}_{2}\right)$, are recursively equivalent. An $L$-algebra $A$ is said to be recursively presented if there is a generating sequence $\bar{a}$ in $A$ such that $\operatorname{diag}_{A}^{+}(\bar{a})$ is recursively enumerable. An $L$-algebra $A$ has solvable word 
problem if there is a generating sequence $\bar{a}$ in $A$ such that $\operatorname{diag}_{A}^{+}(\bar{a})$ is recursive.

Let $T$ be a universal Horn theory and $\mathbf{K}$ the quasi variety axiomatized by $T$. (Note that usually a quasi variety is defined to be the class of models of a set of universal strict Horn sentences; we use the term in a bit more general, rather than standard, sense.) Let $A$ be an algebra in $\mathbf{K}, \bar{a}$ a generating sequence in $A$, and $R \subseteq \operatorname{diag}_{A}^{+}(\bar{a})$. We say that $A$ is defined in $\mathbf{K}$ by the generators $\bar{a}$ and the relations $R$ (or $A$ has a presentation $\langle\bar{a} ; R\rangle$ in $\mathbf{K})$ if $T \cup R \vdash \operatorname{diag}_{A}^{+}(\bar{a})$; in symbols, $A=\langle\bar{a} ; R\rangle_{\mathbf{K}}$, or simply $A=\langle\bar{a} ; R\rangle$. As is well known (see, e.g., Hodges [9]), for every sequence of constants $\bar{c}$ and every set of atomic $L(\bar{c})$-sentences $R$ which has a model in $\mathbf{K}$ there is a (uniquely determined, up to isomorphism over $\bar{c}$ ) $L$-algebra $C$ which has a presentation $\langle\bar{c} ; R\rangle$ in $\mathbf{K}$. If $T$ and $R$ are recursively enumerable, $\operatorname{diag}_{C}^{+}(\bar{c})$ is recursively enumerable too, so $C$ is recursively presented. Moreover, if $R \leq_{e} T$ then $\operatorname{diag}_{C}^{+}(\bar{c}) \leq_{e} T$.

An algebra $A$ is said to be finitely presented in $\mathbf{K}$ if $A=\langle\bar{a} ; R\rangle$ for some tuple $\bar{a}$ and finite $R$. Suppose that $A$ is finitely presented in $\mathbf{K}$ and $\bar{b}$ is an effective sequence in $A$. Then $\operatorname{diag}_{A}^{+}(\bar{b}) \leq_{e} T$. In particular, if $T$ is recursively enumerable then an effectively generated subalgebra (in particular, a finitely generated subalgebra) of an algebra which is finitely presented in $\mathbf{K}$ is recursively presented.

For a recursively enumerable $T$, we say that the Higman Embedding Theorem holds for $\mathbf{K}$ if every recursively presented algebra in $\mathbf{K}$ can be effectively embedded into an algebra which is finitely presented in $\mathbf{K}$.

Let $B$ be an algebra in $\mathbf{K}$. Clearly, $T_{B}=T \cup \operatorname{diag}(B)$ and $T_{B}^{+}=T \cup \operatorname{diag}^{+}(B)$ are universal Horn $L(B)$-theories. Denote by $\mathbf{K}_{B}$ and $\mathbf{K}_{B}^{+}$the quasi varieties axiomatized by $T_{B}$ and $T_{B}^{+}$, respectively. Let $\bar{b}$ list all the elements of $B$. Let $R$ be a set of atomic $L(\bar{a} \bar{b})$-sentences which is consistent with $T_{B}$ and hence with $T_{B}^{+}$. Then the presentation $\langle\bar{a} ; R\rangle$ defines $L(\bar{b})$-algebras $(A, \bar{b})$ and $\left(A^{+}, \bar{b}\right)$ in $K_{B}$ and $K_{B}^{+}$, respectively. Actually they do not differ: $T_{B} \cup R \vdash \psi$ if and only if $T_{B}^{+} \cup R \vdash \psi$ for any atomic $L(\bar{a} \bar{b})$-sentence $\psi$. Indeed, if $T_{B}^{+} \cup R \cup\{\neg \psi\}$ has a model $M$ then $N \times M$ is a model of $T_{B} \cup R \cup\{\neg \psi\}$ for any model $N$ of $T_{B} \cup R$.

We say that an algebra $A$ is finitely presented over its subalgebra $B$ in $\mathbf{K}$ if $(A, \bar{b})$ is finitely presented in $\mathbf{K}_{B}^{+}$or, equivalently, in $\mathbf{K}_{B}$. Suppose that $T$ is recursively enumerable and $B$ is an algebra in $\mathbf{K}$ generated by a tuple $\bar{b}$. Suppose that $A$ is finitely presented over $B$ in $\mathbf{K}$, and $\langle\bar{a} ; R\rangle$ is a finite presentation of $(A, \bar{b})$ in $\mathbf{K}_{B}^{+}$. Then $\operatorname{diag}_{A}^{+}(\bar{a} \bar{b}) \leq_{e} \operatorname{diag}_{A}^{+}(\bar{b})$. Moreover, for any effective sequence $\bar{d}$ in $A$, $\operatorname{diag}_{A}^{+}(\bar{d}) \leq_{e} \operatorname{diag}_{A}^{+}(\bar{b})$. The algebra $A$ is said to be recursively presented over $B$ in $\mathbf{K}$ if $(A, \bar{b})$ has a presentation $\langle\bar{a} ; R\rangle$ in $\mathbf{K}_{B}^{+}$with $R \leq_{e} \operatorname{diag}_{A}^{+}(\bar{b})$, or, equivalently, there is a generating sequence $\bar{d}$ in $A$ such that $\operatorname{diag}_{A}^{+}(\bar{d}) \leq_{e} \operatorname{diag}_{A}^{+}(\bar{b})$. (Note that we needed $B$ to be finitely generated in this definition because otherwise it would depend on the choice of generating sequence in $B$.)

We say that the Generalized Higman Theorem holds for $\mathbf{K}$ if, for every finitely generated algebra $B$ in $\mathbf{K}$, every algebra which is recursively presented over $B$ in $\mathbf{K}$ can be effectively embedded into an algebra which is finitely presented over $B$ in $\mathbf{K}$.

3 Classes of algebras with internal mappings Let $\mathbf{K}$ be a class of $L$-algebras. For a positive integer $n$, we say that $\mathbf{K}$ has the Internal n-ary Mapping Property (the $\mathrm{IMP}_{n}$, for short) if there exists an $L$-term $t_{n}(\bar{x}, \bar{z})$ where $\bar{x}$ is an $n$-tuple of variables such that 
for every algebra $A$ in $\mathbf{K}$ and every mapping $\alpha: A^{n} \rightarrow A$, there are an algebra $C$ in $\mathbf{K}$ containing $A$ and a tuple $\bar{c}$ in $C$ such that $\alpha(\bar{a})=t_{n}^{C}(\bar{a}, \bar{c})$, for every $\bar{a} \in A^{n}$. We say that $\mathbf{K}$ has the effective IMP if it has the $\mathrm{IMP}_{n}$ for every $n$, and the corresponding term $t_{n}$ can be found effectively in $n$.

We say that $\mathbf{K}$ has the Internal Homomorphism Property (the IHP, for short) if, for every positive integer $n$, there exist an $L$-term $h_{n}(x, \bar{z})$ and a finite set of atomic $L$ formulas $S_{n}(\bar{x}, \bar{y}, \bar{z})$ where $\bar{x}=\left(x_{1}, \ldots, x_{n}\right)$ and $\bar{y}=\left(y_{1}, \ldots, y_{n}\right)$ such that for every algebra $A$ in $\mathbf{K}$ and $n$-tuples $\bar{a}=\left(a_{1}, \ldots, a_{n}\right), \bar{b}=\left(b_{1}, \ldots, b_{n}\right)$ in $A$ the following are equivalent.

1. There is a homomorphism from $\langle\bar{a}\rangle$ into $A$ sending $\bar{a}$ to $\bar{b}$.

2. There are an algebra $C$ in $\mathbf{K}$ containing $A$ and a tuple $\bar{c}$ in $C$ such that $S_{n}(\bar{a}, \bar{b}, \bar{c})$ holds in $C$ and $h_{n}^{C}\left(a_{i}, \bar{c}\right)=b_{i}$, for all $i$.

Fact 3.1 (Belegradek [2]) The classes of groups and torsion-free groups have the IHP. One can take the term $\left[x^{u}, v\right]$ as $h_{n}(x, \bar{z})$ and the sets of all $\left[x_{i}^{u}, y_{j}\right]=e$ as $S_{n}(\bar{x}, \bar{y}, \bar{z})$, where $\bar{z}=(u, v)$.

Fact 3.2 (Belyaev [5]) The class of semigroups has the IHP. One can take the term uxv as $h_{n}(x, \bar{z})$ and the sets of all

$$
\begin{aligned}
u x_{i} w x_{j} & =y_{i} u x_{j}, \\
x_{i} x_{j} v & =x_{i} w x_{j} v, \\
x_{i} x_{j} w x_{k} & =x_{i} w x_{j} w x_{k}
\end{aligned}
$$

as $S_{n}(\bar{x}, \bar{y}, \bar{z})$, where $\bar{z}=(u, v, w)$.

Fact 3.3 (Trofimov [15, Belegradek 11) The classes of groups and torsion-free groups have the IMP. One can take the term $\left[x^{u}, w, v\right]$ as $t_{1}(x, \bar{z})$, where $\bar{z}=$ $(u, v, w)$.

Fact 3.4 ([1]) The class of semigroups has the effective IMP. One can take the term $u x_{1} w x_{2}, \ldots, x_{n-1} w x_{n} v$ as $t_{n}(\bar{x}, \bar{z})$, where $\bar{z}=(u, v, w)$.

Theorem 3.5 (cf. [3]) Let $\mathbf{K}$ be a quasi variety of L-algebras which has the JEP. If $\mathbf{K}$ has the IMP $P_{1}$ then $\mathbf{K}$ has the effective IMP.

Proof: If $\mathbf{K}$ is the trivial quasi variety take $x_{1}$ as $t_{n}(\bar{x}, \bar{z})$. Suppose that $\mathbf{K}$ is nontrivial. We explicitly construct $t_{n}$ by induction on $n$. For $n=1$ there is nothing to do. First we construct the term $t_{2}$.

Note that the IMP ${ }_{1}$ implies the existence of a term which is not equivalent in $\mathbf{K}$ to a one-place term. (Indeed, there are $2^{\mu}$ one-place mappings from an infinite algebra of power $\mu$ to itself but using one-place terms one can define at most $\leq|L|+\aleph_{0}$ such mappings.) Let $p\left(y_{1}, \ldots, y_{k}\right)$ be such a term with the minimal possible $k$; clearly $k \geq 2$. We show that one can take the term

$$
t_{1}\left(p\left(y_{1}, \ldots, y_{k-2}, t_{1}\left(x_{1}, \bar{u}\right), t_{1}\left(x_{2}, \bar{v}\right)\right), \bar{w}\right)
$$

as $t_{2}\left(x_{1}, x_{2}, \bar{z}\right)$, where $\bar{z}=\left(y_{1}, \ldots, y_{k-2}, \bar{u}, \bar{v}, \bar{w}\right)$. 
Let $A$ be an algebra in $\mathbf{K}$ and $\rho: A^{2} \rightarrow A$. We must find an algebra $C$ in $\mathbf{K}$ containing $A$ and a tuple $\bar{c}$ in $C$ such that $\rho\left(a_{1}, a_{2}\right)=t_{2}^{C}\left(a_{1}, a_{2}, \bar{c}\right)$, for $a_{1}, a_{2} \in A$. If $|A|=1$, there is nothing to prove. Suppose that $|A|>1$. We can assume $A$ to be infinite because $A$ is embedded into $A^{\omega}$. Suppose $|A|=\mu \geq \aleph_{0}$. Consider an algebra $F$ in $\mathbf{K}$ freely generated by $\left\{b_{1}, \ldots, b_{k-2}, d_{\alpha}, e_{\alpha}: \alpha<\mu\right\}$. Due to the freeness of the generators, if $p^{F}\left(b_{1}, \ldots, b_{k-2}, d_{\alpha}, e_{\beta}\right)=p^{F}\left(b_{1}, \ldots, b_{k-2}, d_{\gamma}, e_{\delta}\right)$ then $(\alpha, \beta)=$ $(\gamma, \delta)$. So the mapping $(x, y) \mapsto p^{F}\left(b_{1}, \ldots, b_{k-2}, x, y\right)$ is injective on $\left\{d_{\alpha}: \alpha<\right.$ $\mu\} \times\left\{e_{\alpha}: \alpha<\mu\right\}$. As $\mathbf{K}$ has the JEP, there is an algebra $B$ in $\mathbf{K}$ containing both $A$ and $F$. Consider bijections $\xi: A \rightarrow\left\{d_{\alpha}: \alpha<\mu\right\}$ and $\sigma: A \rightarrow\left\{e_{\alpha}: \alpha<\mu\right\}$. There are an algebra $D \supseteq B$ in $\mathbf{K}$ and tuples $\bar{r}$ and $\bar{s}$ in $D$ such that $\xi(a)=t_{1}^{D}(a, \bar{r})$ and $\sigma(a)=t_{1}^{D}(a, \bar{s})$, for $a \in A$. So the mapping $\pi: A^{2} \rightarrow A$,

$$
\pi(x, y)=p^{D}\left(b_{1}, \ldots, b_{k-2}, t_{1}^{D}(x, \bar{r}), t_{1}^{D}(y, \bar{s})\right)
$$

is injective. Therefore there is a mapping $v: A \rightarrow A$ such that $\rho=v \circ \pi$. There exist an algebra $C$ in $\mathbf{K}$ containing $D$ and a tuple $\bar{q}$ in $D$ such that $v(a)=t_{1}^{C}(a, \bar{q})$, for $a \in A$. So, for $a_{1}, a_{2} \in A$,

$$
\rho\left(a_{1}, a_{2}\right)=t_{1}^{C}\left(p^{C}\left(b_{1}, \ldots, b_{k-2}, t_{1}^{C}\left(a_{1}, \bar{r}\right), t_{1}^{C}\left(a_{2}, \bar{s}\right)\right), \bar{q}\right)=t_{2}^{C}\left(a_{1}, a_{2}, \bar{c}\right),
$$

where $\bar{c}=\left(b_{1}, \ldots, b_{k-2}, \bar{r}, \bar{s}, \bar{q}\right)$.

Now suppose that $t_{n}$ has been constructed, $n \geq 2$. We show that one can take the term $t_{n}\left(x_{1}, \ldots, x_{n-1}, t_{2}\left(x_{n}, x_{n+1}, \bar{u}\right), \bar{v}\right)$ as $t_{n+1}$. Let $A$ be an algebra in $\mathbf{K}$ and $\alpha: A^{n+1} \rightarrow A$. As above, we can assume $A$ to be infinite. Consider a bijection $\beta$ : $A^{2} \rightarrow A$. Then there is a mapping $\gamma: A^{n} \rightarrow A$ such that

$$
\left.\alpha\left(a_{1}, \ldots, a_{n+1}\right)=\gamma_{(} a_{1}, \ldots, a_{n-1}, \beta\left(a_{n}, a_{n+1}\right)\right),
$$

for $a_{1}, \ldots, a_{n+1} \in A$. There exist an algebra $C \supseteq A$ in $\mathbf{K}$ and tuples $\bar{b}$ and $\bar{d}$ in $C$ such that $\beta\left(a_{1}, a_{2}\right)=t_{2}^{C}\left(a_{1}, a_{2}, \bar{c}\right)$ and $\gamma\left(a_{1}, \ldots, a_{n}\right)=t_{n}^{C}\left(a_{1}, \ldots, a_{n}, \bar{c}\right)$, for $a_{1}, \ldots, a_{n} \in A$. Then for $a_{1}, \ldots, a_{n+1} \in A$,

$$
\alpha\left(a_{1}, \ldots, a_{n+1}\right)=t_{n}^{C}\left(a_{1}, \ldots, a_{n-1}, t_{2}^{C}\left(a_{n}, a_{n+1}, \bar{b}\right), \bar{d}\right)=t_{n+1}^{C}\left(a_{1}, \ldots, a_{n+1}, \bar{c}\right),
$$

where $\bar{c}=(\bar{b}, \bar{d})$, and we are done.

Corollary 3.6 The classes of groups, torsion-free groups, and semigroups have the effective IMP.

It is instructive to write down the term $t_{2}$ for these classes. Here one takes the term $y_{1} y_{2}$ as $p$. In the case of groups or torsion-free groups $t_{1}$ is $\left[x^{u}, w, v\right]$; so the construction gives

$$
\left[\left(\left[x_{1}^{u_{1}}, w_{1}, v_{1}\right]\left[x_{2}^{u_{2}}, w_{2}, v_{2}\right]\right)^{u_{3}}, w_{3}, v_{3}\right]
$$

it is now clear that

$$
\left[\left[x_{1}^{u_{1}}, w_{1}, v_{1}\right]\left[x_{2}^{u_{2}}, w_{2}, v_{2}\right], w_{3}, v_{3}\right]
$$

also fits to be $t_{2}\left(x_{1}, x_{2}, \bar{z}\right)$ where $\bar{z}=\left(u_{1}, u_{2}, v_{1}, v_{2}, w_{1}, w_{2}, w_{3}\right)$. In the case of semigroups the construction leads to the same term as in Fact 3.4. 
Remark 3.7 It is known (cf. [3]) that the following classes do not possess the $\mathrm{IMP}_{1}$ : associative rings, commutative rings, commutative semigroups, an arbitrary nonidentity subclass of the class of locally solvable groups.

Proposition 3.8 Let $\mathbf{K}$ be a recursively axiomatizable quasi variety which has the $I M P_{1}$. Let $A$ be an algebra in $\mathbf{K}$ generated by a sequence $\bar{a}$. Then $A$ can be effectively embedded into a finitely generated algebra $B$ in $\mathbf{K}$ such that, for some tuple $\bar{b}$ generating $B, \operatorname{diag}_{A}^{+}(\bar{a}) \equiv_{e} \operatorname{diag}_{B}^{+}(\bar{b})$.

Proof: Suppose $|A|>1$ (otherwise there is nothing to prove). Let $\bar{a}=\left(a_{i}: i<\right.$ $\omega)$. Consider the algebra $C$ defined in $\mathbf{K}$ by the presentation $\left\langle\bar{a} \bar{c}\right.$; $\left.\operatorname{diag}_{A}^{+}(\bar{a})\right\rangle$, where $\bar{c}=\left(c_{i}: i<\omega\right)$. Clearly, $C \supseteq A$. Since $|A|>1$, in $C$ all $c_{i}$ are distinct. Consider mappings $\alpha$ and $\beta$ from $C$ to $C$ such that $\alpha\left(c_{i}\right)=a_{i}, \beta\left(c_{i}\right)=c_{i+1}$ for all $i$. Applying the IMP ${ }_{1}$ twice, we find an algebra $D$ in $\mathbf{K}$ and tuples $\bar{d}$ and $\bar{e}$ in $D$ such that $\alpha(c)=$ $t_{1}^{D}(c, \bar{d}), \beta(c)=t_{1}^{D}(c, \bar{e})$ for $c \in C$, and in particular, $a_{i}=t_{1}^{D}\left(c_{i}, \bar{d}\right), c_{i+1}=t_{1}^{D}\left(c_{i}, \bar{e}\right)$ for all $i$. So the presentation

$$
\left\langle\bar{a} \bar{c} \bar{d} \bar{e} ; \operatorname{diag}_{A}^{+}(\bar{a}) \cup\left\{a_{i}=t_{1}\left(c_{i}, \bar{d}\right), c_{i+1}=t_{1}\left(c_{i}, \bar{e}\right): i<\omega\right\}\right\rangle
$$

defines in $\mathbf{K}$ an algebra $B$ in which $A$ is naturally embedded. The algebra $B$ is generated by the tuple $\bar{b}=\left(c_{0}, \bar{d}, \bar{e}\right)$; clearly, $\operatorname{diag}_{A}^{+}(\bar{a}) \equiv_{e} \operatorname{diag}_{B}^{+}(\bar{b})$.

\section{An embedding into a relatively finitely presented algebra}

Theorem 4.1 Let $\mathbf{K}$ be a recursively axiomatizable quasi variety of L-algebras. Suppose that $\mathbf{K}$ has the JEP, the IMP $P_{1}$, the IHP, and the Higman Theorem holds for $\mathbf{K}$. Then the Generalized Higman Theorem also holds for $\mathbf{K}$.

Proof: Let $A$ be an algebra in $\mathbf{K}$ generated by a sequence $\bar{a}$. Suppose $A$ is recursively presented in the generators $\bar{a}$ over a tuple $\bar{b}$ in $A$. Since $\operatorname{diag}_{A}^{+}(\bar{a}) \leq_{e} \operatorname{diag}_{A}^{+}(\bar{b})$, there exists a recursively enumerable set $\Theta$ of pairs of the form $(\varepsilon, E)$, where $\varepsilon$ is an atomic $L(\bar{a})$-sentence and $E$ is a finite set of atomic $L(\bar{b})$-sentences such that

$$
\varepsilon \in \operatorname{diag}_{A}^{+}(\bar{a}) \Longleftrightarrow(\varepsilon, E) \in \Theta, \text { for some } E \subseteq \operatorname{diag}_{A}^{+}(\bar{b}) .
$$

Let $\theta=(\varepsilon, E)$ where $\varepsilon$ is $p=q$ and $E$ is $\left\{s_{1}=r_{1}, \ldots, s_{k}=r_{k}\right\}$ for some $L(\bar{a})$ terms $p, q$ and $L(\bar{b})$-terms $s_{i}, r_{i}$. If $k>0$, denote by $\varphi_{\theta}$ and $\psi_{\theta}$ the atomic $L\left(\bar{a} \bar{b} \bar{c}_{\theta}\right)$ sentences $t_{k}\left(s_{1}, \ldots, s_{k}, \bar{c}_{\theta}\right)=p$ and $t_{k}\left(r_{1}, \ldots, r_{k}, \bar{c}_{\theta}\right)=q$, respectively. Here $t_{k}$ is the term which defines in $\mathbf{K} k$-ary mappings; the tuples of new constants $\bar{c}_{\theta}$ are chosen to be pairwise disjoint. If $E=\varnothing$, put $\varphi_{\theta}=\psi_{\theta}=\varepsilon$.

Let $\bar{t}$ be a tuple of $L(\bar{a})$-terms such that $\bar{b}=\bar{t}^{A}(\bar{a})$. Consider an algebra $B$ which is defined in $\mathbf{K}$ by the generators

$$
\bar{a}, \bar{b},\left\{\bar{c}_{\theta}: \theta \in \Theta\right\}
$$

and the relations

$$
\operatorname{diag}_{A}^{+}(\bar{b}) \cup\left\{\bar{b}=\bar{t}^{A}(\bar{a})\right\} \cup\left\{\varphi_{\theta}, \psi_{\theta}: \theta \in \Theta\right\}
$$


We show that $\operatorname{diag}_{A}^{+}(\bar{a})=\operatorname{diag}_{B}^{+}(\bar{a})$, that is, $A$ is naturally embedded into $B$ over the tuple $\bar{b}$.

Indeed, suppose that $\varepsilon \in \operatorname{diag}_{A}^{+}(\bar{a}), \varepsilon$ is $p=q$. Then there is $\theta \in \Theta$ such that $\theta=$ $(\varepsilon, E)$ for some $E \subseteq \operatorname{diag}_{A}^{+}(\bar{b})$. If $E=\varnothing$ then $\varphi_{\theta}=\psi_{\theta}=\varepsilon$ and hence $\varepsilon \in \operatorname{diag}_{B}^{+}(\bar{a})$. If $E$ is $\left\{s_{1}=r_{1}, \ldots, s_{k}=r_{k}\right\}$ then $\varepsilon \in \operatorname{diag}_{B}^{+}(\bar{a})$ because $E \cup\left\{\varphi_{\theta}, \psi_{\theta}\right\}$ holds in $B$. Thus $\operatorname{diag}_{A}^{+}(\bar{a}) \subseteq \operatorname{diag}_{B}^{+}(\bar{a})$.

Suppose $\theta=(\varepsilon, E) \in \Theta$. If $E$ holds in $A$ then $\varepsilon$ holds in $A$. In other words, for the corresponding terms, $s_{1}^{A}=r_{1}^{A}, \ldots, s_{k}^{A}=r_{k}^{A}$ implies $p^{A}=q^{A}$. Then there is a mapping $\alpha: A^{k} \rightarrow A$ such that $\alpha\left(s_{1}^{A}, \ldots, s_{k}^{A}\right)=p^{A}$ and $\alpha\left(r_{1}^{A}, \ldots, r_{k}^{A}\right)=q^{A}$. By choice of the term $t_{k}$, there is an algebra $M_{\theta} \supseteq A$ in $\mathbf{K}$ and a value for $\bar{c}_{\theta}$ in it such that $\varphi_{\theta}, \psi_{\theta}$ hold in $M_{\theta}$. Iterating the construction, we find an algebra $M \supseteq A$ in $\mathbf{K}$ and values for $\bar{c}_{\theta}$ in it such that $\varphi_{\theta}, \psi_{\theta}$ hold in $M$, for all $\theta \in \Theta$. As there is a natural homomorphism from $B$ to $M, \operatorname{diag}_{B}^{+}(\bar{a}) \subseteq \operatorname{diag}_{A}^{+}(\bar{a})$. Thus $\operatorname{diag}_{B}^{+}(\bar{a})=\operatorname{diag}_{A}^{+}(\bar{a})$.

The algebra $B$ is defined in $\mathbf{K}$ by a set of relations which has a form $\operatorname{diag}_{B}^{+}(\bar{b}) \cup P$ where the set $P$ is recursively enumerable. As in the proof of Proposition 3.8. we can effectively embed $B$ into a finitely generated algebra with the same property. Therefore to complete the proof it suffices to prove the following.

Lemma 4.2 Suppose an algebra $C$ is defined in $\mathbf{K}$ by a presentation $\langle\bar{c} ; Q \cup P\rangle$, where $\bar{c}$ is a tuple and $P$ is recursively enumerable. Then there is a presentation $\langle\bar{c} \bar{d} ; Q \cup R\rangle$, where $\bar{d}$ is a tuple and $R$ is finite, such that $C$ is naturally embedded into the algebra $D$ which is defined in $\mathbf{K}$ by this presentation.

Proof: $\quad$ Since the Higman Theorem holds for $\mathbf{K}$, the algebra $\langle\bar{c} ; P\rangle$ can be embedded into an algebra $F=\langle\bar{v} ; V\rangle$ with finite $\bar{v}$ and $V$. Let a tuple of $L(\bar{v})$-terms $\bar{p}$ express the image of $\bar{c}$ under this embedding. As $\mathbf{K}$ has the JEP, the algebras $C$ and $F$ are naturally embedded into $C * F$, the free products of them in $\mathbf{K}$. Let $h_{n}(x, \bar{z})$ and $S_{n}(\bar{x}, \bar{y}, \bar{z})$ be the term and the set of atomic formulas from the definition of the IHP where $n$ is the length of $\bar{c}$. Let $S=S_{n}(\bar{p}, \bar{c}, \bar{z})$ and $H=\left\{h_{n}\left(p_{i}, \bar{z}\right)=c_{i}: i=1, \ldots, n\right\}$. Since there is a homomorphism from $\left\langle\bar{p}^{F}\right\rangle$ onto $\langle\bar{c}\rangle$ which sends $\bar{p}^{F}$ to $\bar{c}$, there exist an algebra $M \supseteq C * F$ in $\mathbf{K}$ and a value for $\bar{z}$ in it such that $S$ and $H$ hold in $M$. So $C * F$ is naturally embedded into

$$
D=\langle\bar{c} \bar{v} \bar{z} ; Q \cup P \cup V \cup S \cup H\rangle_{\mathbf{K}} .
$$

We show that $T \cup V \cup S \cup H \vdash P$. Indeed, let $\psi(\bar{c}) \in P$. Consider an arbitrary model $N$ of $T \cup V \cup S \cup H$. Since $\psi(\bar{p})$ holds in $F$, we have $T \cup V \vdash \psi(\bar{p})$; therefore $\psi(\bar{p})$ holds in $N$. Due to the relations $S \cup H$, there is a homomorphism from $\left\langle\bar{p}^{N}\right\rangle$ onto $\left\langle\bar{c}^{N}\right\rangle$ which sends $\bar{p}^{N}$ to $\bar{c}^{N}$. Therefore $\psi\left(\bar{c}^{N}\right)$ holds in $N$. Thus

$$
D=\langle\bar{c} \bar{v} \bar{z} ; Q \cup V \cup S \cup H\rangle_{\mathbf{K}},
$$

and we can take $\bar{v} \bar{z}$ as $\bar{d}$ and $V \cup S \cup H$ as $R$. The Lemma is proved and the proof of Theorem 4.1 is completed.

Corollary 4.3 For the varieties of groups and semigroups and for the quasi variety of torsion-free groups, the Generalized Higman Theorem holds. 
5 Existentially closed algebras and the $Q$-reducibility Let $A, B \subseteq \omega$. We say that $A$ is $Q$-reducible to $B$ (in symbols, $A \leq_{Q} B$ ) if $A \leq_{e} B$ and there is $\mathcal{F} \subseteq \omega^{2}$ such that $\mathcal{F} \leq_{e} B$ and for every $x \in \omega$,

$$
x \in A \Longleftrightarrow\{y:(x, y) \in \mathcal{F}\} \subseteq B .
$$

Note that for a recursively enumerable $B$, we have $A \leq_{Q} B$ if and only if $A$ is recursively enumerable and there is a recursive function $f$ such that for every $x \in \omega$,

$$
x \in A \Longleftrightarrow W_{f(x)} \subseteq B
$$

It is easy to see that $A \leq_{Q} B$ implies $A \leq_{T} B$; the converse fails even for recursively enumerable $A$ and $B$ (14], Exercise 9-55).

The following result shows how the $Q$-reducibility naturally arises in logic. Let $T$ be a theory of a signature $L$. A diagram type of $T$ is defined to be a set $p(\bar{x})$ of atomic or negated atomic $L$-formulas in variables from a sequence $\bar{x}$ such that

1. $T \cup p(\bar{x})$ is consistent;

2. for every atomic $L$-formula $\varphi(\bar{x})$, either $\varphi(\bar{x}) \in p(\bar{x})$ or $\neg \varphi(\bar{x}) \in p(\bar{x})$.

We denote by $p^{+}$the set of all atomic formulas in $p$.

Theorem 5.1 Let $T$ be a recursively enumerable universal Horn theory of a signature L. Let $q(\bar{x})$ and $p(\bar{y})$ be diagram types of $T$. Suppose that for some recursive sequences of L-terms $\bar{t}$ and $\bar{s}, T \cup q(\bar{s})$ is consistent and $T \cup q(\bar{s}) \vdash p(\bar{t})$. Then $p^{+} \leq Q q^{+}$.

Proof: Suppose the terms in the sequences $\bar{t}$ and $\bar{s}$ are in variables $\bar{u}$. Since $T \cup q(\bar{s})$ is consistent, $q(\bar{s})$ holds in $M=\left\langle\bar{u} ; q^{+}(\bar{s})\right\rangle$. Hence $p(\bar{t})$ holds in $M$ too. Then for any atomic $L$-formula $\psi(\bar{y})$,

$$
\psi \in p^{+} \Longleftrightarrow M \models \psi(\bar{t}) \Longleftrightarrow T \cup q^{+}(\bar{s}) \vdash \psi(\bar{t}) .
$$

Therefore $p^{+} \leq_{e} q^{+}$. Let $\mathcal{F}$ be the set of all pairs $(\psi(\bar{y}), \theta(\bar{x}))$ of atomic $L$-formulas such that $T \cup q^{+}(\bar{s}) \vdash \psi(\bar{t}) \rightarrow \theta(\bar{s})$. Clearly, $\mathcal{F} \leq_{e} q^{+}$. We show that for any atomic $L$-formula $\psi(\bar{y})$,

$$
\psi \in p^{+} \Longleftrightarrow\{\theta:(\psi, \theta) \in \mathcal{F}\} \subseteq q^{+} .
$$

Assume $\psi \in p^{+}$; then $M \models \psi(\bar{t})$. Let $(\psi, \theta) \in \mathcal{F}$. Then $M \models \psi(\bar{t}) \rightarrow \theta(\bar{s})$ and hence $M \models \theta(\bar{s})$. As $M \models q(\bar{s})$, we have $\theta \in q^{+}$. Now assume that $\psi \notin p^{+}$; then $\neg \psi \in p$ and so $T \cup q(\bar{s}) \vdash \neg \psi(\bar{t})$. Hence the theory $T \cup q(\bar{s}) \cup\{\psi(\bar{t})\}$ is inconsistent. Then there is an atomic $L$-formula $\theta(\bar{x}) \notin q^{+}$such that $T \cup q^{+}(\bar{s}) \cup\{\neg \theta(\bar{s}), \psi(\bar{t})\}$ is inconsistent, that is, $T \cup q^{+}(\bar{s}) \vdash \psi(\bar{t}) \rightarrow \theta(\bar{s})$. (Indeed, if for every $\theta(\bar{x}) \notin q^{+}$there were a model $M_{\theta}$ of $T \cup q^{+}(\bar{s}) \cup\{\neg \theta(\bar{s}), \psi(\bar{t})\}$, the Cartesian product of all $M_{\theta}$ would be a model of $T \cup q(\bar{s}) \cup\{\psi(\bar{t})\}$.) So there is $\theta(\bar{x}) \notin q^{+}$such that $(\psi, \theta) \in \mathcal{F}$. The theorem is proved.

We recall that an algebra $A$ in a class of algebras $\mathbf{K}$ is said to be existentially closed in $\mathbf{K}$ if an existential formula over $A$ holds in $A$ if and only if it holds in some algebra in $\mathbf{K}$ containing $A$. 
Theorem 5.2 Let $\mathbf{K}$ be a recursively axiomatizable quasi variety of L-algebras. Suppose that $\mathbf{K}$ has the JEP, the IMP $P_{1}$, the IHP, and the Higman Theorem holds for K. Then for finitely generated algebras $A_{1}$ and $A_{2}$ in $\mathbf{K}$, the following are equivalent:

1. the word problem in $A_{1}$ is $Q$-reducible to the word problem in $A_{2}$;

2. $A_{1}$ is embeddable into every existentially closed algebra in $\mathbf{K}$ containing $A_{2}$.

Proof: $\quad(2) \Rightarrow(1)$ : Let $p_{1}\left(\bar{x}_{1}\right)$ and $p_{2}\left(\bar{x}_{2}\right)$ be the diagram types of some generating tuples in $A_{1}$ and $A_{2}$, respectively. Suppose that condition (1) does not hold, that is, $p_{1}^{+} \Varangle_{Q} p_{2}^{+}$. To construct an existentially closed algebra in $\mathbf{K}$ containing $A_{2}$ but omitting $A_{1}$, it suffices to have the following fact (see [11]).

Fact 5.3 Let the signature $L^{\prime}$ come from $L$ by adding countably many new constants. Let $P$ be a finite set of atomic or negated atomic $L^{\prime}(\bar{c})$-sentences such that $T \cup P \cup p_{2}(\bar{c})$ is consistent. Then for every tuple $\bar{t}$ of $L^{\prime}(\bar{c})$-terms, $T \cup P \cup p_{2}(\bar{c}) \nvdash$ $p_{1}(\bar{t})$.

Fact 5.3 s an application of Theorem 5.1 to the universal Horn theory $T \cup P$; so (2) $\Rightarrow(1)$ has been proved. Consider the following conditions (3) and $\left(3^{+}\right)$.

3. There are an algebra $F \supseteq A_{1}$ which is finitely presented in $\mathbf{K}$ and distinct $u, v \in$ $F$ such that for every homomorphism $\gamma$ from $F$ to an algebra in $\mathbf{K}$, if $\gamma(u) \neq$ $\gamma(v)$ then $\gamma$ is injective on $A_{1}$.

$3^{+}$. There is an algebra $F \supseteq A_{1}, A_{2}$ such that $F$ is finitely presented over $A_{2}$ in $\mathbf{K}$ and every homomorphism from $F$ to an algebra in $\mathbf{K}$ which is injective on $A_{2}$ is injective on $A_{1}$ too.

We prove that each of conditions (1) and (2) is equivalent to (3) in the case $\left|A_{2}\right|=1$, and to $\left(3^{+}\right)$in the case $\left|A_{2}\right|>1$. It remains to prove that, in the case $\left|A_{2}\right|=1$, (1) $\Rightarrow(3) \Rightarrow(2)$ and, in the case $\left|A_{2}\right|>1,(1) \Rightarrow\left(3^{+}\right) \Rightarrow(2)$.

Consider the case $\left|A_{2}\right|=1$. For short, denote $A_{1}$ by $A$. In this case (1) means exactly that $A$ has solvable word problem. First prove (1) $\Rightarrow$ (3). Let a tuple $\bar{a}$ generate $A$. Let $u, v$ be new symbols. For any $L(\bar{a})$-terms $s$ and $r$ with $s^{A} \neq r^{A}$ consider the relations $t_{1}\left(s, \bar{z}_{s r}\right)=u$ and $t_{1}\left(r, \bar{z}_{s r}\right)=v$, where $t_{1}$ is the term from the definition of the Internal Mapping Property. Let $S$ be the set of all these relations. List the members of all tuples $\bar{z}_{r s}$ in a sequence $\bar{z}$. Put

$$
B=\left\langle\bar{a} u v \bar{z} ; \operatorname{diag}_{A}^{+}(\bar{a}) \cup S\right\rangle .
$$

Clearly, $A$ is naturally embedded into $B$. Since $A$ has solvable word problem, the set $\operatorname{diag}_{A}^{+}(\bar{a}) \cup S$ is recursive. Therefore $B$ can be embedded into a finitely presented algebra $F$ in $\mathbf{K}$. It can easily be seen that $F$ satisfies condition (3).

Now we prove (3) $\Rightarrow(2)$. Suppose that the algebra $F$ from (3) has a finite presentation $\langle\bar{x} ; R\rangle$ in $\mathbf{K}$. Let $u=r^{F}, v=s^{F}$ where $r$ and $s$ are $L(\bar{x})$-terms. Let $E$ be an existentially closed algebra in $\mathbf{K}$. Due to the JEP, $E$ is naturally embedded into $E * F$. Therefore there is $\bar{e}$ in $E$ such that $R$ and $r \neq s$ hold in $(E, \bar{e})$. There is a homomorphism $\gamma: F \rightarrow E$ sending $\bar{x}$ to $\bar{e}$. Since $\gamma(u)=r^{E}(\bar{e}) \neq s^{E}(\bar{e})=\gamma(v)$, the homomorphism $\gamma$ is injective on $A$. So $E$ embeds $A$.

Now consider the case $\left|A_{2}\right|>1$. First prove $(1) \Rightarrow\left(3^{+}\right)$. Let tuples $\bar{a}_{1}$ and $\bar{a}_{2}$ generate $A_{1}$ and $A_{2}$, respectively. Since $\operatorname{diag}_{A_{1}}^{+}\left(\bar{a}_{1}\right) \leq_{Q} \operatorname{diag}_{A_{2}}^{+}\left(\bar{a}_{2}\right)$, there is a set $\mathcal{F}$ 
of pairs of the form $(\psi, \theta)$ where $\psi$ is an atomic $L\left(\bar{a}_{1}\right)$-sentence and $\theta$ is an atomic $L\left(\bar{a}_{2}\right)$-sentence such that $\mathcal{F} \leq_{e} \operatorname{diag}_{A_{2}}^{+}\left(\bar{a}_{2}\right)$, and for every atomic $L\left(\bar{a}_{1}\right)$-sentence $\psi$,

$$
\psi \in \operatorname{diag}_{A_{1}}^{+}\left(\bar{a}_{1}\right) \Longleftrightarrow\{\theta:(\psi, \theta) \in \mathcal{F}\} \subseteq \operatorname{diag}_{A_{2}}^{+}\left(\bar{a}_{2}\right)
$$

Let $\tau=(\psi, \theta)$ where $\psi$ is an atomic $L\left(\bar{a}_{1}\right)$-sentence of the form $p=q$ and $\theta$ is an atomic $L\left(\bar{a}_{2}\right)$-sentence of the form $s=r$. Denote by $S$ the set of relations

$$
\left\{t_{1}\left(p, \bar{z}_{\tau}\right)=s, t_{1}\left(q, \bar{z}_{\tau}\right)=r: \tau \in \mathcal{F}\right\}
$$

Clearly, $S \leq_{e} \operatorname{diag}_{A_{2}}^{+}\left(\bar{a}_{2}\right)$. List the members of all tuples $\bar{z}_{\tau}$ in a sequence $\bar{z}$. Since $\mathbf{K}$ satisfies the JEP, $A_{1}$ and $A_{2}$ are naturally embedded into $A_{1} * A_{2}$. Put

$$
B=\left\langle\bar{a}_{1} \bar{a}_{2} \bar{z} ; \operatorname{diag}_{A_{1}}^{+}\left(\bar{a}_{1}\right) \cup \operatorname{diag}_{A_{2}}^{+}\left(\bar{a}_{2}\right) \cup S\right\rangle .
$$

For every $(\psi, \theta) \in \mathcal{F}$, if $\psi$ holds in $A_{1}$ then $\theta$ holds in $A_{2}$. Therefore $A_{1} * A_{2}$ is naturally embedded into $B$. Clearly,

$$
\operatorname{diag}_{A_{1}}^{+}\left(\bar{a}_{1}\right) \cup \operatorname{diag}_{A_{2}}^{+}\left(\bar{a}_{2}\right) \cup S \leq_{e} \operatorname{diag}_{A_{2}}^{+}\left(\bar{a}_{2}\right) .
$$

By Theorem 4.1 the Generalized Higman Theorem holds for $\mathbf{K}$; therefore there exists an algebra $F \supseteq D$ in $\mathbf{K}$ which is finitely presented over $A_{2}$.

We show that $F$ satisfies $\left(3^{+}\right)$. Let $\gamma$ be a homomorphism of $F$ which is not injective on $A_{1}$. Then there is an atomic $L\left(\bar{a}_{1}\right)$-sentence $\psi$ of the form $p=q$ which is not in $\operatorname{diag}_{A_{1}}^{+}\left(\bar{a}_{1}\right)$ but $\gamma\left(p^{F}\right)=\gamma\left(q^{F}\right)$. Then there is an atomic $L\left(\bar{a}_{2}\right)$-sentence $\theta$ of the form $s=r$ which is not in $\operatorname{diag}_{A_{2}}^{+}\left(\bar{a}_{2}\right)$ but $(\psi, \theta) \in \mathcal{F}$. Due to the relations $S$, $\gamma\left(s^{F}\right)=\gamma\left(r^{F}\right)$. So $\gamma$ is not injective on $A_{2}$.

It remains to prove $\left(3^{+}\right) \Rightarrow(2)$. Let $E$ be an existentially closed algebra in $\mathbf{K}$ which embeds $A_{2}$ and let $\bar{a}_{2}^{*}$ realize in $E$ the diagram type of $\bar{a}_{2}$ in $A_{2}$. Suppose that $F$ satisfies $\left(3^{+}\right)$. Then $F$ has a presentation in $\mathbf{K}$ of the form

$$
\left\langle\bar{a}_{2} \bar{x} ; \operatorname{diag}_{A_{2}}^{+}\left(\bar{a}_{2}\right) \cup R\left(\bar{a}_{2}, \bar{x}\right)\right\rangle,
$$

where $\bar{x}$ and $R$ are finite. Due to the JEP, $E$ is naturally embedded into $E * F$. Since $\mathbf{K}$ satisfies the IHP, there exist an algebra $N \supseteq E * F$ in $\mathbf{K}$ and tuples $\bar{c}$ and $\bar{d}$ in $N$ such that

$$
S_{n}\left(\bar{a}_{2}, \bar{a}_{2}^{*}, \bar{c}\right) \cup S_{n}\left(\bar{a}_{2}^{*}, \bar{a}_{2}, \bar{d}\right) \cup\left\{h_{n}\left(\bar{a}_{2}, \bar{c}\right)=\bar{a}_{2}^{*}, h_{n}\left(\bar{a}_{2}^{*}, \bar{d}\right)=\bar{a}_{2}\right\}
$$

holds in $N$ where $n$ is the length of the tuple $\bar{a}_{2}$. Since $E$ is existentially closed, there are $\bar{x}^{\prime}, \bar{a}_{2}^{\prime}, \bar{c}^{\prime}$, and $\bar{d}^{\prime}$ in $E$ such that

$$
R\left(\bar{a}_{2}^{\prime}, \bar{x}^{\prime}\right) \cup S_{n}\left(\bar{a}_{2}^{\prime}, \bar{a}_{2}^{*}, \bar{c}^{\prime}\right) \cup S_{n}\left(\bar{a}_{2}^{*}, \bar{a}_{2}^{\prime}, \bar{d}^{\prime}\right) \cup\left\{h_{n}\left(\bar{a}_{2}^{\prime}, \bar{c}^{\prime}\right)=\bar{a}_{2}^{*}, h_{n}\left(\bar{a}_{2}^{*}, \bar{d}^{\prime}\right)=\bar{a}_{2}^{\prime}\right\}
$$

holds in $E$. Due to $S_{n}\left(\bar{a}_{2}^{*}, \bar{a}_{2}^{\prime}, \bar{d}^{\prime}\right)$ and $h_{n}\left(\bar{a}_{2}^{*}, \bar{d}^{\prime}\right)=\bar{a}_{2}^{\prime}$, there is a homomorphism from $\left\langle\bar{a}_{2}^{*}\right\rangle$ to $\left\langle\bar{a}_{2}^{\prime}\right\rangle$ sending $\bar{a}_{2}^{*}$ to $\bar{a}_{2}^{\prime}$. Therefore $\operatorname{diag}_{A_{2}}^{+}\left(\bar{a}_{2}^{\prime}\right) \cup R\left(\bar{a}_{2}^{\prime}, \bar{x}^{\prime}\right)$ holds in $E$, and so there is a homomorphism $\alpha: F \rightarrow E$ sending $\bar{a}_{2} \bar{x}$ to $\bar{a}_{2}^{\prime} \bar{x}^{\prime}$. Due to $S_{n}\left(\bar{a}_{2}^{\prime}, \bar{a}_{2}^{*}, \bar{c}^{\prime}\right)$ and $h_{n}\left(\bar{a}_{2}^{\prime}, \bar{c}^{\prime}\right)=\bar{a}_{2}^{*}$, there is a homomorphism from $\left\langle\bar{a}_{2}^{\prime}\right\rangle$ to $\left\langle\bar{a}_{2}^{*}\right\rangle$ sending $\bar{a}_{2}^{\prime}$ to $\bar{a}_{2}^{*}$. Therefore $\alpha$ is injective on $A_{2}$ and hence on $A_{1}$. So $A_{1}$ is embedded into $E$. 
Corollary 5.4 Let $\mathbf{K}$ be the variety of groups or the variety of semigroups or the quasi variety of torsion-free groups. Then for finitely generated algebras $A_{1}$ and $A_{2}$ in $\mathbf{K}$, the following are equivalent.

1. The word problem in $A_{1}$ is $Q$-reducible to the word problem in $A_{2}$.

2. $A_{1}$ is embeddable into every existentially closed algebra in $\mathbf{K}$ containing $A_{2}$.

6 Remarks After submitting the paper, I constructed an example which shows that, although the variety of associative rings satisfies the Higman Theorem as the varieties of groups and semigroups do, the situation for associative rings is surprisingly different: there is a finitely generated associative ring which can be omitted in some existentially closed associative ring but still has solvable word problem. That example illustrates well the role of the assumptions of Theorem 5.2. On the other hand, I proved that, for the variety of associative algebras over any field finitely generated over its prime subfield, the Generalized Higman Theorem and the analogue of Theorem 5.2 do hold. The latter results are not special cases of Theorems 4.1 and 5.2. However, it turned out to be possible to isolate certain properties of quasi varieties which, in the first place, hold for the variety of algebras over any field finitely generated over its prime subfield as well as for the varieties of groups and semigroups and, in the second place, guarantee that the Generalized Higman Theorem and the analogue of Theorem 5.2 hold. All of these results are presented in Belegradek [4].

Acknowledgments A part of this work was supported by the Russian Foundation for Basic Research.

\section{REFERENCES}

[1] Belegradek, O. V., "On algebraically closed groups," Algebra and Logic, vol. 13 (1974), pp. 135-143. Zbl 0319.20039IMR 52:2859 1.33.3.3.4

[2] Belegradek, O. V., "Elementary properties of algebraically closed groups," Fundamenta Mathematica, vol. 98 (1978), pp. 83-101.Zbl 0389.20030|MR 57:9530 3.1

[3] Belegradek, O. V., "Classes of algebras with internal maps," pp. 3-10 in Investigations in Theoretical Programming, Kazakh State University, Alma-Ata, 1981.MR 85e:08004 3.5, 3.7

[4] Belegradek, O. V., Model theory of unitriangular and existentially closed groups, D. Sc. Dissertation, Kemerovo State University, Kemerovo, 1995. 6

[5] Belyaev, V. Y., "On algebraically closed semigroups," Siberian Mathematical Journal, vol. 18 (1977), pp. 23-29.ZZbl 0411.20041 3.2

[6] Belyaev, V. Y., "Subrings of finitely presented associative rings," Algebra i Logika, vol. 17 (1978), pp. 627-638.Zbl 0429.16017 MR 81i:16026 1

[7] Belyaev, V. Y., "Embeddability of recursively presented inverse semigroups into finitely presented ones," Sibirskiu Matematicheskil Zhurnal, vol. 25 (1984), pp. 50-54. Zbl 0545.20047|MR 85m:20083 1

[8] Higman, G., "Subgroups of finitely presented groups," Proceedings of the Royal Society of London, Ser. A, vol. 262 (1961), pp. 455-474. Zbl 0104.021011MR 24:A152 
[9] Hodges, W., Model theory, Cambridge University Press, Cambridge, 1993. Zbl 0789.03031||MR 94e:03002 2

[10] Kukin, G. P., "Subalgebras of recursively presented Lie algebras," Algebra i Logika, vol. 18 (1979), pp. 311-327.

[11] Macintyre, A., "Omitting quantifier-free types in generic structures," The Journal of Symbolic Logic, vol. 37 (1972), pp. 512-520. Zbl 0273.02038|MR 49:37, 1,5

[12] Murski1, V. L., "Isomorphic embeddability of a semigroup with enumerable set of defining relations to a finitely presented semigroup," Matematicheskie zametki, vol. 1 (1967), pp. 217-224. MR 35:1686 1

[13] Neumann, B. H., "The isomorphism problem for algebraically closed groups," pp. 553562 in Word Problems, edited by W. W. Boone et al., North-Holland, Amsterdam, 1973. Zbl 0198.34103|MR 54:2767 1

[14] Rogers, H., Theory of recursive functions and effective computability, McGraw-Hill, New York, 1967. Zbl 0183.01401MR 88b:03059 1. 2.5

[15] Trofimov, M. Y., "On definability in algebraically closed systems," Algebra and Logic, vol. 13 (1974), pp. 198-202.Zbl 0373.02035 MR 55:2559 3.3

[16] Ziegler, M, “Algebraisch abgeschlossene Gruppen,” pp. 449-576 in Word Problems II, edited by S. I. Adian et al., North-Holland, Amsterdam, 1980.Zbl 0451.20001 MR 82b:20004 1.11

\author{
Kemerovo State University \\ Kemerovo 650043 \\ RUSSIA \\ email: beleg@kaskad.uucp.stanet.ru
}

